\title{
Integrated cognitive and physical fitness training enhances attention abilities in older adults
}

Joaquin Anguera ( $\nabla$ joaquin.anguera@ucsf.edu )

UCSF

Joshua Volponi

UCSF

Alexander Simon

UCSF

Courtney Gallen

UCSF

Camarin Rolle

UCSF

Roger Anguera-Singla

UCSF

Christian Thompson

USF

Adam Gazzaley

UCSF

Article

Keywords: aging, attention, cognitive training, physical training, EEG

Posted Date: December 17th, 2021

DOI: https://doi.org/10.21203/rs.3.rs-1171873/v1

License: (c) (i) This work is licensed under a Creative Commons Attribution 4.0 International License.

Read Full License 
Integrated cognitive and physical fitness training enhances attention abilities in older adults

Joaquin A. Anguera*1,2,3,4 , Joshua J. Volponi ${ }^{1,2,3}$, Alexander J. Simon ${ }^{1,2,3}$, Courtney L. Gallen ${ }^{1,2,3}$, Camarin E. Rolle $^{1,2,3}$, Roger Anguera-Singla ${ }^{1,2,3}$, Christian J. Thompson ${ }^{6}$, Gazzaley, Adam*1,2,3,4,5.

\section{Author Affiliation:}

1. Neuroscape, University of California San Francisco, San Francisco, CA 94158

2. Department of Neurology, University of California San Francisco, San Francisco, CA 94158

3. Weill Institute for Neurosciences \& Kavli Institute for Fundamental Neuroscience, University of California San Francisco, San Francisco, CA 94158

4. Department of Psychiatry, University of California San Francisco, San Francisco, CA 94158

5. Department of Physiology, University of California San Francisco, San Francisco, CA 94158

6. Department of Kinesiology, University of San Francisco, San Francisco, CA

* Corresponding Authors:

Joaquin A. Anguera, Adam Gazzaley

Neuroscape Center

Sandler Neurosciences Center, Room 502

675 Nelson Rising Lane

San Francisco, CA 94158

Joaquin.anguera@ucsf.edu, Adam.gazzaley@ucsf.edu

Key Words: aging, attention, cognitive training, physical training, EEG 


\begin{abstract}
Preserving attention abilities is of great concern to older adults who are motivated to maintain their quality of life as they age. Both cognitive and physical fitness interventions have been utilized in intervention studies to assess maintenance and enhancement of attention abilities in seniors, and a coupling of these approaches is a compelling strategy to buttress both cognitive and physical health in a time- and resource-effective manner. With this perspective, we created a closed-loop, motion-capture video game (Body-Brain Trainer: BBT) that adapts a player's cognitive and physical demands in an integrated approach, thus creating a personalized and cohesive experience across both domains. Older adults who engaged in two months of BBT improved on both physical fitness and attention outcome measures beyond that of an expectancy-matched, active, placebo control group, with maintenance of improved attention performance evidenced 1 year later. Following training, the BBT group's improvement on the attention outcome measure exceeded performance levels attained by an untrained group of 20-year-olds, and showed age-equilibration of a neural signature of attention shown to decline with age: midline frontal theta power. These findings highlight the potential benefits of an integrated, cognitive-physical, closed-loop training platform as a powerful tool for both cognitive and physical enhancement in older adults.
\end{abstract}




\section{Introduction}

Over the last decade there has been a surge of research aimed at improving cognitive abilities by harnessing neuroplasticity via cognitive training ${ }^{1-5}$. The augmentation of deficient attention abilities is especially of interest for older adults, given well documented age-related declines in these abilities ${ }^{6-14}$, which are exacerbated in the presence of interference ${ }^{2,7,15-17}$. Cognitive training as a whole has come under warranted scrutiny given consistent shortcomings in study design and outcomes: i) minimal extension of benefits beyond the training interventions themselves, ii) lack of appropriate control groups, and iii) absence of follow-up testing to assess sustainability of observed effects ${ }^{18-20}$. However, there have been several notable examples of cognitive interventions enhancing non-trained cognitive abilities in older adults ${ }^{10,21-23}$, with these findings aligning with systemic reviews supporting the utility of cognitive interventions in the older adult population ${ }^{24-26}$.

In contrast to the uncertainty surrounding the benefits of cognitive training, the utility of physical fitness interventions for older adults' general health has been well established. Fitness-based interventions have also been shown to benefit cognitive control abilities ${ }^{27-32}$, with such findings hinting at the possibility that combining cognitive and physical training approaches may lead to greater cognitive benefits than either approach alone (although not supported to date ${ }^{33-36}$ ). The use of combined cognitive and physical interventions are especially compelling as a strategy to maintain both cognitive and physical health ${ }^{37}$, given that such a pairing can be a time- and resource-effective approach for addressing multiple risk factors in older adults ${ }^{38}$. Indeed, combined training has been shown to result in greater participant enjoyment compared to either sequential training ${ }^{39}$ or physical exercise alone ${ }^{40}$.

We have previously demonstrated that cognitive interventions using closed-loop, adaptive algorithms ${ }^{41}$ designed to target specific cognitive abilities in older adults can improve task performance and neural measures of cognition ${ }^{12,23,42-44}$. These interventions, delivered as engaging video games, have shown positive effects not only with older adults, but in diverse populations ${ }^{45-48}$. Here we created a novel cognitive intervention for older adults (Body-Brain Trainer, BBT; Figure 1) that requires participants to perform full-body, physical movements in response to cognitive challenges that engage different cognitive control domains ${ }^{41,49}$. As in our previous work ${ }^{42,50,51}$, we integrated real-time adaptivity using closed loop mechanics for each cognitive ability being challenged. Given the movement demands of BBT, we also implemented an adaptive physical fitness challenge by using real-time heart rate data to 
titrate game play for a personalized and integrated training experience across both cognitive and physical domains (see Methods and Supplementary Materials for more details on the BBT software).

In the present study, we sought to evaluate the primary question of whether BBT could improve measures of attention and physical fitness in older adults beyond that of an expectancy-matched, active placebo control group. We also interrogated the following secondary questions: what is the neural mechanism underlying positive cognitive effects, do any observed cognitive improvements persist one year later without booster training sessions, does BBT result in older adults achieving comparable levels to young adults on our primary outcome measure, and does this intervention affect other measures of cognitive control (working memory).

\section{RESULTS}

\section{Participation and Study Overview:}

To interrogate the posed questions, we performed a double-blinded, randomized, placebo-controlled study (registered on clinicaltrials.gov [NCT03032796]) where 49 healthy older adults (OA; mean age $=68.5+/-6.3,26$ females) were randomly assigned to an intervention group (BBT: $n=24)$ or an active, expectancy-matched control group (Mind-Body Trainer (MBT): $n=25$, see Supplementary Figure 1 and Methods for details). A total of 41 of these participants (BBT $=21, M B T=20$ ) returned 1-year after completing their intervention to assess for the presence of sustained cognitive benefits (a CONSORT figure describing participant enrollment can be found at Supplementary Figure 2). Details regarding improvements on the BBT training intervention itself can also be found in the Supplementary Materials, including Supplementary Figures 3-6.)

\section{Sustained Attention:}

Here we used a vigilance task as our primary cognitive outcome measure to assess the intervention's impact on attention abilities. This task was a customized Continuous Performance Task (CPT), which is a modified version of a well-validated sustained attention task, the Test of Variables of Attention (TOVA) ${ }^{52}$, used as an outcome measure in our previous intervention studies ${ }^{42,48,53}$ (Figure 2a). As our metric of interest, we focused on the ex-gaussian tau of response times, a non-parametric measure of distribution skewing that quantifies attentional lapses by examining the distribution of long response times. Several studies have demonstrated a clear advantage of this measure over traditional measures of response time (although we report on traditional metrics of response time and response time variance in the Supplemental Materials) given that it explains a greater proportion of variance without needing to 
employ data trimming techniques ${ }^{54-56}$. Importantly, this measure has been shown to reflect performance inconsistencies that are especially present in both healthy older adults and those with mild cognitive impairment ${ }^{57}$, and has been used to support neural noise $e^{58}$ and dedifferentiation ${ }^{59-61}$ theories of cognitive aging.

Using a repeated measures ANOVA to evaluate change in CPT performance from pre- to postintervention (see 'Statistical methods' in Methods), we found a significant time (Pre, Post) by intervention (BBT, MBT) interaction $\left(F_{1,45}=7.49, p=0.009\right.$, Cohen's $d=.79$ ), with performance improvements (i.e. a reduction in tau from pre to post) in the BBT group ( $\Delta=+15.73$ msec improvement, $\left.t_{23}=2.29, p=0.03\right)$, but not in the MBT group ( $\Delta=-6.46$ msec improvement, $t_{24}=-1.60, p=0.13$; Figure 2b, and Table 1 for values). When this measure was compared to a separate untrained cohort of young adults ( $n=51$; Figure 3) who completed this task in a single visit, the BBT group did not show expected age-related performance differences at baseline; i.e., the BBT group exhibited equivalent performance to young adults $\left(t_{73}=.42, p=\right.$ .67; see Table 1 for values). However, following training, the BBT group showed significantly lower tau (i.e., better performance) than young adults $\left(t_{74}=2.20, p=.03\right)$. Finally, in evaluating stability of performance gains over time, follow-up tests 1-year later revealed that neither group showed significant differences from post-training performance levels (BBT: $t_{20}=1.61, p=.12 ; M B T: t_{19}=.34 p=.74$; see Table 1).

We also performed an exploratory analysis of attention (not part of pretrial registration assessments) to assess how a more challenging complex visual discrimination task with varying levels of distraction would be impacted by these interventions (see Supplementary Figure 7a and Methods for task details). The same pattern of results as described for CPT in terms of both the statistical interaction and follow-up tests 1-year later were observed on the complex visual discrimination task (see Supplemental Materials and Supplementary Figure 7b).

\section{Neural Correlates of Sustained Attention:}

In addition to measuring cognitive performance, participants also underwent electroencephalography (EEG) recordings during the CPT task to examine neural mechanisms that underlie performance changes from pre to post (i.e., improved ability to stabilize their attention as revealed by reductions in tau). Here we utilized the neural metric of midline frontal theta power, given that it has been associated with sustained attention abilities ${ }^{62,63}$ and is a sensitive marker of changes in attention abilities following interventions ${ }^{42,45,64}$. A repeated measures ANOVA revealed a significant group by time interaction $\left(F_{(1,43)}=6.61, p=0.014\right.$, Cohen's $d=.74$; Figure $\left.2 c\right)$, with post-hoc paired-samples t-tests showing a significant increase in power following the intervention for the BBT group $\left(t_{23}=-2.77, p=0.011\right)$, but not 
in the control group $\left(t_{20}=0.79, p=0.44\right)$. Once again, the same pattern of results was also observed for the complex visual discrimination task using comparable EEG analyses (see Supplemental Materials and Supplementary Figure 7c).

When compared to the group of young adults, the older adult BBT group showed expected agerelated reductions in pre-training theta power $\left(t_{56}=3.10, p=.003\right)$. After the intervention period, the BBT group's post-training power increased to a level equivalent to that of young adults $\left(t_{57}=.11, p=.91\right.$, see Figure 3, and Table 1 for values). With respect to assessing the stability of these changes 1-year later, neither group showed significant differences from their post-training levels (BBT: $t_{18}=1.16, p=.26$; MBT: $t_{14}=.95, p=.36$; see Table 1 for values).

\section{Fitness Measures}

We assessed whether the BBT intervention led to measurable improvements in physical fitness measures beyond that of the control group by focusing on metrics of balance ${ }^{65-69}$ and blood pressure $\mathrm{e}^{70-}$

${ }^{73}$, given their association with real-world health outcomes in older adults. Using a repeated measures ANOVA to test for differential group improvement over time, we observed a significant group by time interaction on a balance measure (i.e., the limits of stability test associated with risk of falling ${ }^{74,75}$; $F_{(1,44)}=8.37, p=0.006$, Cohen's $d=.90$, Figure $\left.4 a\right)$; notably, the BBT group significantly improved on this stability measure $\left(t_{21}=-3.81, p=0.001\right)$ whereas the MBT group did not $\left(t_{23}=-0.81, p=0.94\right)$. With respect to blood pressure, a repeated measures ANOVA revealed a significant group by time interaction of diastolic blood pressure $\left(F_{(1,41)}=9.32, p=0.004\right.$, Cohen's $d=.78$, Figure $\left.4 b\right)$, with the BBT group significantly decreasing their diastolic blood pressure after training $\left(t_{21}=4.35, p=0.001\right)$ while the MBT group did not $\left(t_{20}=0.68, p=0.51\right)$. See Methods and Supplementary Materials for details on systolic blood pressure as well as other exploratory physical fitness metrics.

\section{Working Memory and Basic Response Time Assessments}

We also explored whether BBT led to an improvement on a working memory task (described in trial registration as a secondary outcome) that has been shown to improve in previous intervention studies of older adults ${ }^{16,42,46,76}$ (see Methods for task details). A repeated measures ANOVA of tau using a withinsubject factor of Session (Pre, Post) and a between-group factor of Study Group (BBT, MBT) did not demonstrate a Study Group x Time interaction for any of the three working memory conditions (no distractions, ignore distractions, attend to distractors), indicating that tau on this working memory test did not differentially change between study groups $\left(F_{(1,39)} \leqq 0.95, p \geqq .34\right.$, see Table 1 for no distractor 
condition values). Similarly, independent t-test analyses showed that there was no group difference in performance post-training on any of the three conditions $\left(t_{(42)} \leqq 1.46, p \geqq 0.52\right)$.

Finally, we administered a Basic Response Time (BRT) task as a measure of simple motoric response time to ensure that any differences observed between groups were not due to improvements in basic motoric speed (see Methods for task details). A repeated measures ANOVA with a within-subject factor of Session (Pre, Post) and a between-group factor of Study Group (BBT, Control) did not reveal an interaction, indicating that RT did not differentially change as a function of training between study groups $\left(F_{(1,40)}=2.16, p=.15\right.$, see Table 1 for values). Similarly, there was no RT performance difference posttraining between the BBT and MBT groups $\left(t_{(42)}=.44, p=.67\right)$. This same analysis approach revealed the same outcome for RTV, with no observed interaction $\left(F_{(1,40)}=.31, p=.58\right)$ or difference post-training $\left(t_{(42)}=\right.$ $1.5, p=.13$ ). These results suggest that differences found on the primary outcome measures cannot be attributed to improvements in basic motoric speed or motoric variability.

\section{DISCUSSION}

\section{Overview:}

Here we demonstrate that an integrated cognitive-physical fitness intervention (BBT) improved measures of physical fitness, as well as objective measures of attention beyond an expectancy-matched, active placebo control group. After the intervention, their post-training cognitive performance reached comparable, and in one case superior, levels to younger adults, with these gains being maintained 1 year later. Here we discuss the implications of these findings and how they relate to other efforts aimed at improving cognitive function in older adults.

\section{Physical, Cognitive, and Neural Enhancements:}

Given the movement demands of $\mathrm{BBT}$, it was unsurprising yet reassuring that engagement led to improved balance, consistent with results from other exergaming studies in older adults ${ }^{77,78}$. However, this is the first study of its kind that also evidenced diastolic blood pressure improvements beyond a control group, suggesting that the adaptive fitness mechanics spurred a physiological benefit not previously seen before. BBT also had a positive effect on attention abilities in older adults, in alignment with other combined cognitive-physical intervention studies that evidenced enhanced attention in older adults $^{33,35,79}$. However, the benefits observed here were attained with a significantly shorter training regimen ( 8 weeks) compared to the majority of these other combined studies (typically between 12-16 weeks, with some lasting up to 30 weeks), and with much less logistical burden by the training in each 
domain being integrated rather than split across different training days. Another key difference from previous studies was the incorporation of adaptivity, not just for cognitive challenges but also for physical fitness demands, which allowed us to titrate the physical training appropriately to each individual's fitness level. We hypothesize that the integration of the cognitive and physical training within a video game experience may have spurred the observed benefits after a relatively abbreviated intervention time.

The attention-based improvements reported here are comparable to those found in other aging intervention studies from our group that utilized different closed-loop adaptive mechanics to improve attention both with ${ }^{23}$ and without distraction ${ }^{42}$. Attention improvements documented using the same CPT task have also been observed in a recent meditation-based intervention study in younger adults ${ }^{48}$. This convergence of attention improvements emerging from different types of closed-loop interventions in older adults and other populations is particularly compelling ${ }^{46,80-82}$. Indeed, the results from these studies suggest the potential for individuals to select their preferred digital treatments without concerns about differential efficacy.

While there is ample evidence that combined interventions in older adults can have positive effects on overall cognitive function ${ }^{83-86}$, few studies have included neuroimaging assessments to examine the underlying mechanisms of improvements observed ${ }^{36,87,88}$. Studies involving such combined approaches have reported increased cerebral glucose metabolism in frontal and sensorimotor regions ${ }^{36}$, enhanced $\mathrm{fMRI}$ resting state functional connectivity between frontal and temporal regions ${ }^{88}$, and increased $f M R I$ resting state synchronization at temporal and cerebellar regions ${ }^{87}$. These findings highlight distinct neural metrics involving 'task-free' measures across different brain regions associated with improved cognitive performance, as brain-behavioral correlations were observed in each study. Here, we observed that the activation of a frontal top-down network accompanied improved attentional performance without external distraction, as well as increased trial-by-trial neural consistency that corresponded with improved attentional focus when distractions were present. These findings are aligned with our previous work in older adults evidencing similar top-down theta power enhancements following a closed-loop video game intervention (NeuroRacer) ${ }^{42}$, as well as improved theta coherence following a distraction training intervention in both older humans and rats ${ }^{23}$. Thus, empirical support exists for the idea that midline frontal theta is an especially sensitive marker of attentional control changes in intervention studies ${ }^{64}$.

Comparisons to young adults and long-term maintenance of benefits: 
The present findings offer a mixed interpretation of whether the observed attention-based improvements were a remediation of age-related deficiencies or the intervention enhanced nondeficient processes. The older adult BBT cohort exhibited age-related deficits in midline frontal theta power that increased to levels equivalent to young adults after training, unlike the control group. However, the BBT cohort showed comparable performance levels at baseline (CPT tau) to the young adults, although after training they were significantly better than their younger counterparts. Previous studies have evidenced age-related declines on these same metrics ${ }^{42,89}$. The current results support a compensation effect ${ }^{90,91}$, given that improvements in the BBT group led to performance levels exceeding that of younger adults and suggest that integrated cognitive and physical approaches designed to augment plasticity in neural systems may have the potential to remediate certain aging deficits ${ }^{23,92}$.

Other work has suggested that combined interventions may have a particular advantage with respect to long-term maintenance of observed benefits ${ }^{86}$. We offer evidence here demonstrating that each group had comparable performance levels at the 1-year mark compared to levels attained immediately post-training. These findings should be considered with caution given that very few intervention studies targeting cognitive control abilities have evidenced persisting effects well past the initial treatment period ${ }^{93-100}$. Indeed, our own work failed to realize the persistence of the more distant transfer effects several years later ${ }^{64}$, and even here the sensitivity of these measures one year later is unclear. While it has been argued that the value of these types of interventions are dubious without the demonstration of sustained benefits beyond the initial treatment period ${ }^{101,102}$, we posit that these findings better highlight the potential utility of incorporating additional booster sessions for sustaining benefits.

\section{Conclusions:}

Here we observed that BBT led to improvement in the ability of healthy older adults to stabilize their attention from moment to moment on a vigilance task beyond that of an expectancy-matched, placebo control group. Beyond the methodological approach used here, these results are highly relevant for older adults given the steady advancement of innovative approaches designed to help these individuals with their cognition and physical fitness. The present findings are consistent with recent meta-analyses highlighting the positive effects of combined interventions on cognitive function $83,86,103,104$, with accompanying physical benefits supporting the use of such technology to realize meaningful benefit in both cognitive and physical domains. Thus, comparable combined cognitive and physical interventions may be a viable time- and resource-effective approach for older adults to maintain (or even enhance) both their cognitive and physical health. 


\section{Limitations:}

There are several limitations with the present study that are important to note. First, this work does not directly interrogate whether BBT leads to beneficial effects beyond those achieved with training cognitive or physical abilities in isolation. Several studies and meta-analyses have already interrogated this question ${ }^{33-36}$, with these works suggesting that the combined approach does not appear to yield synergistic effects beyond cognitive training alone. Furthermore, due to the experimental design, we cannot directly assess how much of the observed attention improvements can be attributed to the individual physical and cognitive training components incorporated in BBT. Indeed, both cognitive interventions and physical fitness training have been shown to induce attention improvements and positive functional changes in the prefrontal cortex (i.e. increased task-based activations) ${ }^{99,105,106}$ in line with present findings. Our goal here was to assess if there was value in an integrated approach beyond that of an expectancy-matched placebo control group, as a positive result sets the stage for a future trial directly examining the mechanistic contributions of training each domain in isolation.

Second, while we observed improvements on distinct measures of attention, as in previous work, improvements did not emerge on a working memory outcome measure as it did in previous intervention-based studies ${ }^{42,46}$ (see Supplementary Materials). One possible explanation for this result is that BBT disproportionately engaged attention-based circuitry during training, given that both working memory ${ }^{107}$ and cognitive flexibility abilities ${ }^{108,109}$ call upon attentional resources for their successful utilization. Thus, attention abilities were engaged during each BBT module across the entire intervention periods. This interpretation, while speculative, is consistent with previous work that targeted cognitive flexibility and observed improvements on the same outcome measures used here (CPT and the working memory task), as well as on the same neural measures ${ }^{42}$.

\section{METHODS}

\section{Participants:}

The study was approved by the Committee on Human Research at the University of California, San Francisco, and registered on clinicaltrials.gov [NCT03032796]. The present study directly examined the intervention and expectancy matched placebo arms described in the registration, with the collection of the two mechanistic control arms planned for a future RCT. 49 healthy, older adult (OA) individuals consented to participate in this study. OAs were randomly assigned to a training group (body-brain trainer; $\mathrm{BBT} ; \mathrm{N}=24$, mean age $=68.8+/-5.9,13$ females) or an active, expectancy-matched control group 
(mind-body trainer; $\mathrm{MBT} ; \mathrm{N}=25$, mean age $=68.20+/-6.75,13$ females). All participants were from the San Francisco Bay Area and recruited through online and newspaper advertisements. All participants had normal or corrected-to-normal vision, had no history of stroke, traumatic brain injury, or psychiatric illness, were not taking psychotropic, hormonal, or cardiovascular medications, and did not have any physical or mental conditions that may interfere with their daily activities (e.g., migraine headaches, substance abuse, neuropathy; see Supplemental Materials regarding our neuropsychological assessment protocol). All participants reported playing less than 2-hours of video games per month. All participants gave written informed consent, and were paid $\$ 15 /$ hour for their in-lab and at-home participation.

\section{Study Design:}

For those participants randomized to the BBT group, they were asked to come to Neuroscape at UCSF 3 days per week, 1 hour per visit (36min of on-task training time per session, 24 minutes allocated for warm up/cool down/breaks) for 8 weeks ( 24 hours total, 14.4 hours of actual training time). Each of these visits were accompanied by an onsite trainer to facilitate the training experience for the participant and ensure that training was being completed in a safe manner. For those participants randomized to the MBT group, they were loaned an iPad tablet (9.7 inch screen size; 1024 x 768 screen resolution) for their training session following their 'Pre-training' assessment, and were instructed to train with their assigned task at home for six weeks, 5-days per week, with 30-minute training sessions per day, for a maximum of thirty 30 -minute training sessions ( 15 hours of training total). All MBT participants were instructed to train sitting down with the tablet on a flat surface, such as a table, in a location with minimal external distraction.

We used a study design that involved several layers of blinding: i) data was collected by a group of research associates who were blinded to the identity of the intervention group, ii) data analysis (which was anonymized to conceal intervention group identity) was performed by a different group of researchers, and iii) participants were blinded to the group assignment following randomization procedure, which was done prior to the first participant visit to the lab. Blinding began at the point of recruitment, where all participants were informed that they were being recruited for a study designed to test the efficacy of software interventions for improving cognition in a variety of domains. Neither the BBT nor the MBT participants were aware of the other group or the task that they trained with. Both groups were administered the same instructions and brief overview of the goals of the study, namely to determine if the training game could improve cognitive abilities. Thus, all participants were told that they were part of an active intervention to improve their cognitive abilities (see below for details on 
how we established matched expectancy $\left.{ }^{110}\right)$. Finally, one study coordinator was informed of the treatment assignments, as their role was solely to provide technical and other support during the training.

All participants reported to our UCSF Neuroscape laboratories prior to training ('Pre-training' session) and following the completion ('Post-training' session) of training (1-week grace period from start/end of training) to compete a battery of cognitive and physical outcome measures to assess training-related changes. Participants were invited to return to the laboratory 1 year after their posttraining outcome assessment to evidence which, if any, positive training effects persisted in the cognitive domain. Over the course of the study, 3 participants in the BBT group and 2 participants in the MBT group voluntarily withdrew from the study, resulting in the complete pre- and post-training data sets from 24 BBT participants and 25 MBT participants. One additional participant had a non-training related adverse event which caused them to withdraw from the study prior to be randomized to a group. We were unable to collect EEG data from three participants at pre-training due to equipment malfunctions, and, due to technical issues with photodiodes, we were unable to time-lock the EEG to the event onsets for several other participants ( $n=4$ at pre-training, $n=4$ at post-training). There was one participant who did not return for their post physical assessment.

\section{Intervention Descriptions:}

BBT paradigm. BBT is comprised of three modules, with each targeting a different aspect of cognitive control: visual search tasks for attention (with increasing distraction), spatial span/multiple object tracking tasks for working memory, and a task-switching paradigm targeting goal management/cognitive flexibility abilities. There are also three different tasks with ascending difficulty within each module, such that advancing to the next level engages a fresh challenge while maintaining interest (for example, a change from a spatial span condition to a multiple object tracking condition with working memory demands). Comparable to our previous work using cognitive measures alone ${ }^{42,50,51}$, here we integrate real-time adaptivity for both the cognitive and physical aspects of the gameplay. For each cognitive task, difficulty scales on a trial-by-trial basis, with a correct trial performed within a thresholding-determined response window leading to shorter response window by $10 \mathrm{msec}$, and an incorrect trial leading to a lengthening of the response window by $30 \mathrm{msec}$ (thus, a 1"up"/3"down" staircase). These cognitive adaptive algorithms are designed to assure participants remain at an $\sim 80 \%$ rate of accuracy, a level that is not too easy nor too hard, so that it is enjoyable and engaging. For the physical training, difficulty is tied to the demands associated with the distance an individual must travel for a given response and the amount of time allocated to complete this response. These movement-related aspects are directly 
responsive to whether heart rate is below/within/above a predetermined heart rate window to ensure a moderately intense workout that does not impede the ability to perform the cognitive task. For example, if one is playing the game below their assigned heart rate range, the software will automatically increase the distance that the participant must move to respond with their hands/feet on each trial until their heart rate is within the specified range. Training sessions are linked, such that the next session begins at the level attained at the end of the previous session. Participants are provided two types of feedback: 1) real-time feedback - indicating whether the participant successfully detected or classified the target and 2) punctuated feedback - participants advance through a series of "levels" that are reported at the beginning and end of each run. We describe each module in greater detail in the Supplementary Materials, as well as the pre-training calibration procedures undertaken to personalize the experience for each participant.

\section{MBT battery:}

To mitigate any potential placebo effects brought on by participant expectations, we identified a suitable active control condition based on participant predictions of potential training-related gains as in our previous work ${ }^{48}$. We identified a set of three commercially available iOS apps (Supplementary Figure 1) that were matched to our BBT program in terms of expectation of improvement on our cognitive outcome measures (see below for description of the statistical selection process). Those apps were a language learning app (Duolingo; www.duolingo.com), a Tai Chi app (Tai Chi Step by Step; www.imoblife.net), and a logic games app (100 Logic Games; www.andreasabbatini.com/LogicGames.aspx). For Duolingo, participants were given a choice of which language they wanted to learn from those available on the app. Within the app, we set a 10 min training time per day. During training, the app takes users through a series of modules that increase in difficulty and are only unlocked sequentially following completion of an earlier module. Modules are organized topically (e.g., Food, Animals, Phrases, etc.) and each module contains listening, speaking, vocabulary, and translation tasks and culminates with a topic quiz. At the end of each lesson the app provides a progress report showing learning "streaks" and the accumulation of "lingots" (Duolingo currency). These feedback features are meant to keep participants motivated. For Tai Chi, users simply open the app and select from a series of modules that provide detailed and easy-to-follow instructions on how to perform many basic, intermediate, and advanced Tai Chi movements and is geared toward beginners with no Tai Chi experience. Each description can be read or listened to and is accompanied by an animation. Users were instructed to then practice the exercise themselves several times after each lesson. The logic games app is comprised of a series of "puzzle sets" that revolve around 
a particular theme and which get progressively more difficult as people advance. The puzzles are similar to the more well-known Sudoku puzzles, but provide a more engaging experience with colorful icons, unique rule sets for each theme, and increasing difficulty. For each puzzle, users are given a task (e.g., plant trees according to specific rules), a time limit, and several hints that they can unlock. Participants were instructed to spend approximately $10 \mathrm{~min}$ with each app each training day ( 5 days per week for 6 weeks). For Duolingo, the time was set internally in the app. For the other two apps, participants selftimed their training, and they recorded their time on a training log. All participants completed the MBT intervention at home using an iPad Mini 2 (iOS version 8.2; Apple) that was supplied by the UCSF Neuroscape Center. On the day they were given their iPad to begin training, participants were also provided an instructional binder with instructions on how to play, a calendar for recording notes and comments throughout their training experience, and were given e-mail support throughout the intervention period. Throughout the intervention period, technical support for the iPads and all software was provided via e-mail, phone, and in-person contact, when needed.

\section{Behavioral Cognitive Control Assessments:}

Attention without distraction. A custom continuous performance task (CPT) designed in Presentation (http://neurobs.com) was used to assess vigilance without distraction. This tool is a modified version of a well-validated vigilance task (Figure 2a), the Test of Variables of Attention (TOVA), that we have used as an outcome measure in previous intervention studies. For the present study, we adapted the task for use with EEG recordings, which requires many trials with an actual response, and also facilitates the examination of ex-gaussian tau, a non-parametric measure of distribution skewing of response times ${ }^{111-}$ 114. A key factor that led to our decision to use this particular metric stems from recent work demonstrating that the relationship between behavioral variability and inattention is driven by the tail of the ex-Gaussian distribution of response times (which is indexed by tau) rather than variability in the normal distribution component ${ }^{115}$. In this task, participants maintain fixation on a central crosshairs and grey squares are shown on a black background at the top or bottom of the field of view. Stimuli are presented frequently at the top of the screen as a 4:1 ratio of targets to nontargets and participants are instructed to only respond to these stimuli. Participants completed 2 blocks of 125 trials with 100 targets per block, yielding 200 total targets and 50 non-target trials.

Working memory fidelity task. We used a delayed recognition working memory paradigm designed to measure changes in participants' ability to maintain an accurate mental representation of items in working memory either in presence or absence of distracting or interfering information. We have used 
versions of this task in numerous previous studies ${ }^{16,42,46,76}$. To summarize briefly, this paradigm consisted of three different conditions that were presented in blocks: 1) no distraction (ND), 2) Ignore distractor (ID, distractor was present, but participants were informed that the distractor was to be ignored), and 3) Attend Distractor ( $A D$, participants were required to make a judgment about the interfering stimulus). Each run was preceded by an instruction slide informing the participant which condition they would be performing. Each trial began with the presentation of a face displayed for $800 \mathrm{~ms}$, followed by a delay period ( $3 \mathrm{~s})$, the presentation of a face stimulus as a distractor in the ID and AD conditions ( $800 \mathrm{~ms}$ ), a second delay period $(3 \mathrm{~s})$, and the presentation of a face probe $(1 \mathrm{~s})$. The participants were instructed to make a match/nonmatch button press response at the probe as quickly as possible, without sacrificing accuracy. This was followed by a self-paced intertrial interval (ITI). The experiment was programmed in E-Prime (https://pstnet.com/products/e-prime/) and the stimuli were presented on a CRT monitor.

Basic response time task. Here we administered a basic response time (BRT) task as a measure of simple motoric response time to ensure that any differences observed between groups were not due to differences in basic motoric quickness. Basic motor speed was assessed in the form of a simple targetdetection task during their pre-training assessment, to ensure that any training-related enhancements in performance were not attributed to a general motoric speed increase, but rather to enhancements in cognitive control processes. On this task participants pressed a keyboard button as quickly as possible upon the appearance of a circle at the center of the screen (50 trials). Average response time (the time between the target appearance and button press) was assayed as a unit of basic motor speed ${ }^{116}$.

Complex visual discrimination amid distractions. For this exploratory measure of attention in the presence of variable distraction (considered exploratory given that it was not described in our clinicaltrial.gov submission), we used a custom Filter Task ${ }^{117}$ (Supplemental Figure 7a) to assess how well participants were able to identify targets in the presence of task-irrelevant information ${ }^{48,118,119}$. The experiment was programmed in MATLAB's psychophysics toolbox (http://psychtoolbox.org/) and the stimuli were presented on a CRT monitor. In this task, participants must attend to an array of different numbers of items (either 1 or 3 red rectangles) with or without the presence of 2 visual distractors ( 2 blue or green rectangles): set size 1 no distractors ( 1 total item), set size 1 with distractors ( 3 total items), set size 3 no distractors ( 3 total items), set size 3 with distractors ( 5 total items). Half of each of the trials for each condition began with a cue indicating the participant should attend to either the left or the right side of the screen. The procedure for each trial began with a $750 \mathrm{~ms}$ fixation cross following by a right/left cue $(200 \mathrm{~ms})$ and then a $300 \mathrm{~ms}$ blank ISI. Next, a sample set from one of the four 
conditions was shown for $200 \mathrm{~ms}$ followed by a 900 ms blank delay and then a probe set containing the same number of red rectangles as in the sample in either the same orientation or with a single rectangle of altered orientation ( $50 \%$ of each). The probe screen remained visible until participants responded with a "Yes" or "No" button press indicating whether or not one of the attended rectangles changed orientation. Participants completed 8 blocks of 80 trials, yielding 160 trials per condition. Participants were instructed to respond as fast as possible without sacrificing accuracy. As in our previous work ${ }^{48}$, we focused on Response Time Variability (RTV) as our measure of interest here.

\section{Neural Cognitive Control Assessments:}

EEG Recordings. Neurophysiological data were recorded during each cognitive control assessment using an active two head cap (Cortech Solutions) with a BioSemiActiveTwo 64-channel EEG acquisition system in conjunction with BioSemiActiView software (Cortech Solutions). Signals were amplified and digitized at $1024 \mathrm{~Hz}$ with a 16-bit resolution. Anti-aliasing filters were used and data were band-pass filtered between 0.01-100 Hz during data acquisition. For each EEG recording session, a $1 \times 1$-inch white box was flashed for $10 \mathrm{~ms}$ at one of the corners on the stimulus presentation monitor at the start of each trial. A photodiode (http://www.gtec.at/Products/Hardware-and-Accessories/g.TRIGbox-Specs-Features) captured this change in luminance to facilitate precise time-locking of the neural activity associated with each sign event. During the experiment, these corners were covered with tape to prevent participants from being distracted by the flashing light.

EEG Preprocessing. Preprocessing was conducted using the EEGLAB software ${ }^{120}$. Noisy channels were identified upon initial visual inspection, were removed from the data, and interpolated using a spherical spline interpolation, using the average signal of the surrounding channels to reconstruct the data in the removed channel. The data were then down-sampled to $1024 \mathrm{~Hz}$ to reduce the computational demand without losing any important information in the data. A finite impulse response filter with a high-pass cutoff of $1 \mathrm{~Hz}$ was applied to remove drift, and then a low-pass filter at $40 \mathrm{~Hz}$ was applied to remove high frequency noise. Ocular correction was performed by using ICA to isolate and remove activity induced by eye-blinks and lateral eye movements from the signal. The data were then re-referenced to the average signal of all channels. Epochs of $-1000 \mathrm{~ms}$ to $+1000 \mathrm{~ms}$ were generated for each stimulus type for subsequent analyses. Epochs containing excessive peak-to-peak deflections $( \pm 100 \mu \mathrm{V})$ were removed. 
Midline Frontal Theta Analyses. Midline frontal theta $(4-7 \mathrm{~Hz})$ power ( $\mathrm{mft}$ Power) has been implicated in sustained attention abilities ${ }^{42,121-123}$, including correlating with RTV across the lifespan ${ }^{124}$, that can evidence the engagement of the prefrontal cognitive control processes during a given task. This particular marker has also demonstrated a level of sensitivity in revealing changes following a digital intervention in multiple studies $23,42,45,48,64$. Time series were created by resolving $4-40 \mathrm{~Hz}$ activity using a fast Fourier transform (FFT) in EEGLAB in epochs from -1000 to +1000 msec. Midline frontal theta total power analyses (evoked power + induced power) across trials from the vigilance task was conducted by resolving $4-40 \mathrm{~Hz}$ activity using a fast Fourier transform in EEGLAB, with these values subsequently referenced to a -900 to -700 pre-stimulus baseline (thus relative power $(\mathrm{dB})$ ). After $\mathrm{mft}$ relative power was computed, we specifically interrogated a predetermined cluster of frontal electrodes ( $F z, F P z, A F 3, A F 4$, and $A F z$ ) at the time window of maximum power, as this is the same approach that we have previously utilized in several intervention studies interrogating $\mathrm{mft}$ power changes $\mathrm{s}^{42,45,48,64}$.

\section{Physical outcome measures:}

To capture changes in physical fitness due to training during the study, all participants underwent a comprehensive physical outcomes assessment in addition to the cognitive assessments described above. Our targeted outcome measures were a senior-specific measure of stability indicative of fall risk ${ }^{74,75}$ as well as a physiological measure of fitness and health (diastolic blood pressure) ${ }^{72}$. Our decision to focus on diastolic (rather than systolic) blood pressure was based on diastolic blood pressure being a proportionally larger contributor to mean arterial pressure (MAP) $)^{125}$, with MAP being an important predictor of cardiovascular disease $\mathrm{e}^{126-128}$. Descriptions of each exploratory fitness measure evaluated, including systolic blood pressure, are described in the Supplemental Materials; note that these measures came from the Senior Fitness Test (SFT) to assess global physical performance ${ }^{129}$.

\section{Training-related expectancy ratings:}

In an effort to identify an expectancy matched placebo versus the BBT intervention, we first compared expectancy on 10 different apps to BBT in a sample of 261 participants (Female=135, mean age=33.5). Each participant was randomly assigned to view a video of BBT or one of 10 potential apps ( $n=15-30$ individuals per app) which we did not hypothesize would improve cognitive abilities. They were then asked to rate the extent to which the presented intervention would lead to improvements on these outcomes, using a Likert scale measuring 1 (no improvement) to 7 (a lot of improvement). Following this first round of testing, we selected the three apps that had the highest expectancy matching scores, and subsequently collected new expectancy data in a sample of 76 young adults to 
assess the level of expectancy involving the combination of these three apps $(n=37)$ versus BBT $(n=39)$. Here we found no differences in participant expectations of improvement on our CPT task $\left(t_{74}=0.24, P=\right.$ $0.62,95 \% \mathrm{Cl}:-1.3$ to 0.77 ) or on our filter task ( $\mathrm{t}_{74}=0.55, \mathrm{P}=0.16,95 \% \mathrm{Cl}:-0.30$ to 1.8$)$, suggesting that we had comparable expectancy on this battery of control apps as compared to the BBT intervention with respect to our cognitive control outcome measures.

To determine if these expectancy findings held with older adults, we had 91 older adults complete this same expectancy survey. These older adults were shown a recording of either the BBT $(n=46$, mean age $=64.4$, Female $=28)$ or the MBT $(n=45$, mean age $=63.2$, Female $=31)$ interventions and subsequently shown a video describing our cognitive outcome measures. We observed no significant difference between interventions in terms of participant expectations of improvement on the CPT $\left(t_{89}=-\right.$ 1.84, $\mathrm{p}=0.069$, with the MBT intervention showing a trend towards having greater expectation of improving on this measure than the BBT group) and the Filter tasks ( $\mathrm{t}_{89}=-0.682, \mathrm{p}=0.50$ ). To ensure that these findings extended to the present study, we asked all BBT and MBT participants to complete a comparable version of this survey after they had completed their first few days of training on their assigned intervention to see if their experience led to different expectancy-based perceptions. This was motivated especially by the fact that the MBT training experience was distinct from the BBT training experience in that they completed their training at home on a tablet by themselves, whereas the BBT group trained at a laboratory on a large screen TV in the presence of a trainer. Once again, we observed no significant difference between interventions in terms of participant expectations of improvement on the CPT ( $t_{36}=1.26, p=0.21, M B T$ mean=5.0, BBT mean=5.4) and the Filter tasks $\left(t_{36}=0.64, p=0.53, M B T\right.$ mean=5.0, BBT mean=5.3).

\section{Statistical Analysis Approach:}

Analyses were conducted by researchers who were blind to group membership. To test for training effects on each of the collected outcome measures, we used an repeated measures ANOVA approach as in our previous work ${ }^{42,53}$. Statistically, no between-group differences were observed for at baseline involving: (1) age $\left(t_{47}=0.35, p=0.73\right) ;(2)$ gender $\left(t_{47}=-0.15, p=0.88\right) ;(3)$ CPT performance $\left(t_{45}=1.84, p=.07\right) ;(4)$ Filter task performance $\left(t_{46}=1.34, p=.19\right) ;(5)$ mft power $\left(t_{44}=1.70, p=.10\right)$; $(6)$ mft ITC $\left(t_{41}=1.11, p=.28\right)$; (7) limits of stability $\left(\mathrm{t}_{44}=1.05, \mathrm{p}=.30\right)$; (8) diastolic blood pressure $\left(\mathrm{t}_{43}=1.77, \mathrm{p}=.08\right)$. For those measures that showed a trend towards a group difference at baseline (CPT performance, mft power, and blood pressure), we performed an analysis of covariance (ANCOVA) with post-training performance as the dependent variable, pre-training performance as the covariate, and group as the fixed factor. Our reasoning for using this approach is that this analysis accounts for variation around the post-test means 
that arises from the variation where participants began at pretest ${ }^{130}$. Using this approach, we observed a nearly significant group difference following training for the CPT task $\left(F_{(1,46}=3.85, p=0.056\right)$, a significant group effect for $\mathrm{mft}$ power $\left(\mathrm{F}_{(1,44}=4.35, \mathrm{p}=0.043\right)$ and a significant group effect for diastolic blood pressure $\left(F_{(1,42}=6.93, p=0.012\right)$, supporting the assertion that the reported ANOVA effects were not driven by trending baseline differences between groups. For post-hoc analysis of the within-group changes, we performed two-tailed, paired-sample t-tests on each group separately to test for significant differences between each testing session. We report Cohen's $d$ for all significant $(p<.05)$ and trending $(p<.10)$ ANOVA results, using the Hedges and Olkin correction ${ }^{131}$ for small sample bias.

\section{Acknowledgements:}

A special thanks to J. Souza and E. Kong for help with data analyses, Q. Coppola, B. Hernandez, K.

Samplay, B. Larkin, S. Fenton, A. Halili, G. Lara, J. Manalang, C. Janzen, A. Cailles, B. Luu, L. Muñoz, K. Lee, A. Holt, and C. Matlock (and all other student trainers from the University of San Francisco's Kinesiology Department) for their help in collecting data for this study. We would also like to thank S. Niblett for help with study coordination. Thanks to all of our participants whose time and efforts made this work possible. This study was funded by the generous support of our Neuroscape donors (Neuroscape Network), who provided us the freedom to undertake and complete this project.

\section{Author Contributions:}

J.A.A. and A.G. designed the experiments; J.A.A., R.A.S., C.R. and A.G. developed the BBT software; J.V., A.S., C.G., C.T. collected the data; J.A.A., J.V., A.S., C.G., and C.T. analyzed the data; and J.A.A., J.V., A.S., and A.G. wrote the paper. All authors discussed the results.

\section{Competing Interests statement:}

A.G. is a co-founder, shareholder, BOD member, and advisor for Akili Interactive Lab, a company that produces therapeutic video games. 


\section{References}

1 Boot, W. R. et al. Video games as a means to reduce age-related cognitive decline: attitudes, compliance, and effectiveness. Front Psychol 4, 31, doi:10.3389/fpsyg.2013.00031 (2013).

2 Mishra, J., Anguera, J. A., Ziegler, D. A. \& Gazzaley, A. A cognitive framework for understanding and improving interference resolution in the brain. Prog Brain Res 207, 351-377, doi:10.1016/B978-0-444-63327-9.00013-8 (2013).

3 Berry, A. S., Zanto, T. P., Rutman, A. M., Clapp, W. C. \& Gazzaley, A. Practice-Related Improvement in Working Memory is Modulated by Changes in Processing External Interference. J. Neurophysiol. 102, 1779-1789, doi:10.1152/jn.00179.2009 (2009).

4 Taubert, M., Villringer, A. \& Ragert, P. Learning-Related Gray and White Matter Changes in Humans: An Update. Neuroscientist 18, 320-325, doi:Doi 10.1177/1073858411419048 (2012).

5 Ceccarelli, A. et al. Cognitive learning is associated with gray matter changes in healthy human individuals: A tensor-based morphometry study. Neuroimage 48, 585-589, doi:Doi 10.1016/J.Neuroimage.2009.07.009 (2009).

6 Anguera, J. A., Reuter-Lorenz, P. A., Willingham, D. T. \& Seidler, R. D. Failure to engage spatial working memory contributes to age-related declines in visuomotor learning. J Cogn Neurosci 23, 11-25, doi:10.1162/jocn.2010.21451 (2011).

$7 \quad$ Clapp, W. C. \& Gazzaley, A. Distinct mechanisms for the impact of distraction and interruption on working memory in aging. Neurobiol Aging, doi:S0197-4580(10)00042-4 [pii]

10.1016/j.neurobiolaging.2010.01.012 (2010).

8 Gazzaley, A., Sheridan, M. A., Cooney, J. W. \& D'Esposito, M. Age-related deficits in component processes of working memory. Neuropsychology 21, 532-539, doi:200712685-002 [pii]

10.1037/0894-4105.21.5.532 (2007).

9 Grady, C. L. et al. Age-related changes in regional cerebral blood flow during working memory for faces. Neuroimage 8, 409-425, doi:S1053-8119(98)90376-7 [pii]

10.1006/nimg.1998.0376 (1998).

10 Rolle, C. E., Anguera, J. A., Skinner, S. N., Voytek, B. \& Gazzaley, A. Enhancing Spatial Attention and Working Memory in Younger and Older Adults. Journal of Cognitive Neuroscience 29, 1483-1497, doi:10.1162/jocn_a_01159 (2017).

11 Zanto, T. P., Toy, B. \& Gazzaley, A. Delays in neural processing during working memory encoding in normal aging. Neuropsychologia 48, 13-25, doi:S0028-3932(09)00321-2 [pii]

10.1016/j.neuropsychologia.2009.08.003 (2010).

12 Cohn, N. B., Dustman, R. E. \& Bradford, D. C. Age-related decrements in Stroop Color Test performance. J Clin Psychol 40, 1244-1250, doi:10.1002/10974679(198409)40:5<1244::aid-jclp2270400521>3.0.co;2-d (1984).

13 Comalli, P. E., Jr., Wapner, S. \& Werner, H. Interference effects of Stroop color-word test in childhood, adulthood, and aging. J Genet Psychol 100, 47-53, doi:10.1080/00221325.1962.10533572 (1962).

14 Klein, M., Ponds, R. W., Houx, P. J. \& Jolles, J. Effect of test duration on age-related differences in Stroop interference. J Clin Exp Neuropsychol 19, 77-82, doi:10.1080/01688639708403838 (1997).

15 Clapp, W. C., Rubens, M. T. \& Gazzaley, A. Mechanisms of working memory disruption by external interference. Cereb Cortex 20, 859-872, doi:bhp150 [pii]

10.1093/cercor/bhp150 (2010).

16 Clapp, W. C., Rubens, M. T., Sabharwal, J. \& Gazzaley, A. Deficit in switching between functional brain networks underlies the impact of multitasking on working memory in older adults. Proc Natl Acad Sci U S A 108, 7212-7217 (2011). 
17 Fernandes, M. A., Pacurar, A., Moscovitch, M. \& Grady, C. Neural correlates of auditory recognition under full and divided attention in younger and older adults.

Neuropsychologia 44, 2452-2464, doi:S0028-3932(06)00154-0 [pii]

10.1016/j.neuropsychologia.2006.04.020 (2006).

18 Sala, G., Tatlidil, K. S. \& Gobet, F. Video game training does not enhance cognitive ability: A comprehensive meta-analytic investigation. Psychol Bull 144, 111-139, doi:10.1037/bul0000139 (2018).

19 Simons, D. J. et al. Do "Brain-Training" Programs Work? Psychol Sci Public Interest 17, 103-186, doi:10.1177/1529100616661983 (2016).

20 Owen, A. M. et al. Putting brain training to the test. Nature 465, 775-778, doi:nature09042 [pii]

10.1038/nature09042 (2010).

21 Anguera, J. A. et al. in Society for Neuroscience.

22 Karbach, J., Mang, S. \& Kray, J. Transfer of task-switching training in older age: the role of verbal processes. Psychol Aging 25, 677-683, doi:2010-16183-001 [pii]

10.1037/a0019845 (2010).

23 Mishra, J., de Villers-Sidani, E., Merzenich, M. \& Gazzaley, A. Adaptive training diminishes distractibility in aging across species. Neuron 84, 1091-1103, doi:10.1016/j.neuron.2014.10.034 (2014).

24 Lampit, A., Hallock, H. \& Valenzuela, M. Computerized cognitive training in cognitively healthy older adults: a systematic review and meta-analysis of effect modifiers. PLoS medicine 11, e1001756, doi:10.1371/journal.pmed.1001756 (2014).

25 Gavelin, H. M., Lampit, A., Hallock, H., Sabates, J. \& Bahar-Fuchs, A. CognitionOriented Treatments for Older Adults: a Systematic Overview of Systematic Reviews. Neuropsychol Rev 30, 167-193, doi:10.1007/s11065-020-09434-8 (2020).

26 Hill, N. T. et al. Computerized Cognitive Training in Older Adults With Mild Cognitive Impairment or Dementia: A Systematic Review and Meta-Analysis. Am J Psychiatry 174, 329-340, doi:10.1176/appi.ajp.2016.16030360 (2017).

27 Pan, C.-Y. et al. Effects of Physical Exercise Intervention on Motor Skills and Executive Functions in Children With ADHD. Journal of Attention Disorders, 1087054715569282, doi:10.1177/1087054715569282 (2015).

28 Neudecker, C., Mewes, N., Reimers, A. K. \& Woll, A. Exercise Interventions in Children and Adolescents With ADHD. Journal of Attention Disorders, 1087054715584053, doi:10.1177/1087054715584053 (2015).

29 Halperin, J. M., Berwid, O. G. \& O'Neill, S. Healthy Body, Healthy Mind?: The Effectiveness of Physical Activity to Treat ADHD in Children. Child and Adolescent Psychiatric Clinics of North America 23, 899-936, doi:https://doi.org/10.1016/i.chc.2014.05.005 (2014).

30 Chang, Y.-K., Liu, S., Yu, H.-H. \& Lee, Y.-H. Effect of Acute Exercise on Executive Function in Children with Attention Deficit Hyperactivity Disorder. Archives of Clinical Neuropsychology 27, 225-237, doi:10.1093/arclin/acr094 (2012).

31 Berwid, O. G. \& Halperin, J. M. Emerging Support for a Role of Exercise in AttentionDeficit/Hyperactivity Disorder Intervention Planning. Current Psychiatry Reports 14, 543551, doi:10.1007/s11920-012-0297-4 (2012).

32 Archer, T. \& Kostrzewa, R. M. Physical Exercise Alleviates ADHD Symptoms: Regional Deficits and Development Trajectory. Neurotoxicity Research 21, 195-209, doi:10.1007/s12640-011-9260-0 (2012).

33 Desjardins-Crepeau, L. et al. Effects of combined physical and cognitive training on fitness and neuropsychological outcomes in healthy older adults. Clin Interv Aging 11, 1287-1299, doi:10.2147/cia.s115711 (2016). 
34 Guo, W. et al. Effect of Combined Physical and Cognitive Interventions on Executive Functions in OLDER Adults: A Meta-Analysis of Outcomes. Int J Environ Res Public Health 17, doi:10.3390/ijerph17176166 (2020).

35 Shatil, E. Does combined cognitive training and physical activity training enhance cognitive abilities more than either alone? A four-condition randomized controlled trial among healthy older adults. Front Aging Neurosci 5, 8, doi:10.3389/fnagi.2013.00008 (2013).

36 Shah, T. et al. A combination of physical activity and computerized brain training improves verbal memory and increases cerebral glucose metabolism in the elderly. Transl Psychiatry 4, e487, doi:10.1038/tp.2014.122 (2014).

37 Yang, C. et al. Effectiveness of Combined Cognitive and Physical Interventions to Enhance Functioning in Older Adults With Mild Cognitive Impairment: A Systematic Review of Randomized Controlled Trials. Gerontologist 60, 633-642, doi:10.1093/geront/gnz149 (2020).

38 Gavelin, H. M. et al. Combined physical and cognitive training for older adults with and without cognitive impairment: A systematic review and network meta-analysis of randomized controlled trials. medRxiv, 2020.2008.2008.20170654, doi:10.1101/2020.08.08.20170654 (2020).

39 McEwen, S. C. et al. Simultaneous Aerobic Exercise and Memory Training Program in Older Adults with Subjective Memory Impairments. J Alzheimers Dis 62, 795-806, doi:10.3233/JAD-170846 (2018).

40 Eggenberger, P., Schumacher, V., Angst, M., Theill, N. \& de Bruin, E. D. Does multicomponent physical exercise with simultaneous cognitive training boost cognitive performance in older adults? A 6-month randomized controlled trial with a 1-year followup. Clin Interv Aging 10, 1335-1349, doi:10.2147/CIA.S87732 (2015).

41 Mishra, J., Anguera, J. A. \& Gazzaley, A. Video Games for Neuro-Cognitive Optimization. Neuron 90, 214-218, doi:10.1016/j.neuron.2016.04.010 (2016).

42 Anguera, J. A. et al. Video game training enhances cognitive control in older adults. Nature 501, 97-101, doi:nature12486 [pii]

10.1038/nature12486 [doi] (2013).

43 Wais, P. E., Arioli, M., Anguera-Singla, R. \& Gazzaley, A. Virtual reality video game improves high-fidelity memory in older adults. Sci Rep 11, 2552, doi:10.1038/s41598021-82109-3 (2021).

44 Berry, A. S. et al. The influence of perceptual training on working memory in older adults. PLoS ONE 5, e11537, doi:10.1371/journal.pone.0011537 (2010).

45 Anguera, J. A. et al. A pilot study to determine the feasibility of enhancing cognitive abilities in children with sensory processing dysfunction. Plos One 12, doi:ARTN e0172616

10.1371/journal.pone.0172616 (2017).

46 Anguera, J. A., Gunning, F. M. \& Arean, P. A. Improving late life depression and cognitive control through the use of therapeutic video game technology: A proof-ofconcept randomized trial. Depress Anxiety, doi:10.1002/da.22588 (2017).

47 Arean, P. A. et al. The Use and Effectiveness of Mobile Apps for Depression: Results From a Fully Remote Clinical Trial. J Med Internet Res 18, e330, doi:10.2196/jmir.6482 (2016).

48 Ziegler, D. A. et al. Closed-loop digital meditation improves sustained attention in young adults. Nat Hum Behav 3, 746-757, doi:10.1038/s41562-019-0611-9 (2019).

49 Anguera, J. A. \& Gazzaley, A. Video games, cognitive exercises, and the enhancement of cognitive abilities. Current Opinion in Behavioral Sciences 4, 160-165 (2015).

50 Mishra, J. \& Gazzaley, A. Cross-species approaches to cognitive neuroplasticity research. Neuroimage, doi:10.1016/j.neuroimage.2015.09.002 (2015). 
51 Mishra, J. \& Gazzaley, A. Closed-loop cognition: the next frontier arrives. Trends Cogn Sci 19, 242-243, doi:10.1016/j.tics.2015.03.008 (2015).

52 Greenberg, L. M. T.O.V.A. Continuous Performance Test Manual., (The TOVA Company, 1996).

53 Mishra, J., de Villers-Sidani, E., Merzenich, M. \& Gazzaley, A. Adaptive training diminishes distractibility in aging across species. Neuron. 84, 1091-1103. doi: 1010.1016/j.neuron.2014.1010.1034. Epub 2014 Nov 1020. (2014).

54 Moret-Tatay, C. et al. The Role of Feedback on Learning: Evidence Through ExGaussian Components. Procedia - Social and Behavioral Sciences 191, 2578-2581 (2015).

55 Whelan, R. Effective analysis of reaction time data. The Psychological Record 58, 475482 (2008).

56 van Belle, J. et al. Capturing the dynamics of response variability in the brain in ADHD. Neuroimage Clin 7, 132-141, doi:10.1016/j.nicl.2014.11.014 (2015).

57 Gorus, E., De Raedt, R., Lambert, M., Lemper, J. C. \& Mets, T. Reaction times and performance variability in normal aging, mild cognitive impairment, and Alzheimer's disease. J Geriatr Psychiatry Neurol 21, 204-218, doi:21/3/204 [pii]

10.1177/0891988708320973 (2008).

58 Voytek, B. et al. Age-Related Changes in 1/f Neural Electrophysiological Noise. The Journal of Neuroscience 35, 13257-13265, doi:10.1523/JNEUROSCI.2332-14.2015 (2015).

59 Li, S.-C., Lindenberger, U. \& Sikström, S. Aging cognition: from neuromodulation to representation. Trends in Cognitive Sciences 5, 479-486, doi:http://dx.doi.org/10.1016/S1364-6613(00)01769-1 (2001).

60 Li, S.-C. \& Lindenberger, U. in Cognitive neuroscience of memory (ed L. G. Nilsson H. J. Markowitsch) 103-146 (Hogrefe \& Huber Publishers, 1999).

61 Hülür, G., Ram, N., Willis, S. L., Schaie, K. W. \& Gerstorf, D. Cognitive dedifferentiation with increasing age and proximity of death: Within-person evidence from the Seattle Longitudinal Study. Psychology and Aging 30, 311-323, doi:10.1037/a0039260 (2015).

62 Nigbur, R., Ivanova, G. \& Sturmer, B. Theta power as a marker for cognitive interference. Clin Neurophysiol 122, 2185-2194, doi:S1388-2457(11)00255-0 [pii]

10.1016/j.clinph.2011.03.030 (2011).

63 Cavanagh, J. F. \& Frank, M. J. Frontal theta as a mechanism for cognitive control. Trends Cogn Sci 18, 414-421, doi:10.1016/j.tics.2014.04.012 (2014).

64 Anguera, J. A. et al. Long-term maintenance of multitasking abilities following video game training in older adults. Neurobiol Aging 103, 22-30, doi:10.1016/j.neurobiolaging.2021.02.023 (2021).

65 Carande-Kulis, V., Stevens, J. A., Florence, C. S., Beattie, B. L. \& Arias, I. A cost-benefit analysis of three older adult fall prevention interventions. J Safety Res 52, 65-70, doi:10.1016/j.jsr.2014.12.007 (2015).

66 Karlsson, L. et al. Outcomes of a Physical Therapist-Led, Statewide, Community-Based Fall Risk Screening. J Geriatr Phys Ther 43, 185-193, doi:10.1519/JPT.0000000000000228 (2020).

67 Noonan, R. K., Sleet, D. A. \& Stevens, J. A. Closing the gap: a research agenda to accelerate the adoption and effective use of proven older adult fall prevention strategies. J Safety Res 42, 427-430, doi:10.1016/j.jsr.2010.12.002 (2011).

68 Renfro, M. O. \& Fehrer, S. Multifactorial screening for fall risk in community-dwelling older adults in the primary care office: development of the fall risk assessment \& screening tool. J Geriatr Phys Ther 34, 174-183, doi:10.1519/JPT.0b013e31820e4855 (2011). 
69 Sleet, D. A., Moffett, D. B. \& Stevens, J. CDC's research portfolio in older adult fall prevention: a review of progress, 1985-2005, and future research directions. J Safety Res 39, 259-267, doi:10.1016/j.jsr.2008.05.003 (2008).

70 Dai, X. et al. Cardiovascular physiology in the older adults. J Geriatr Cardiol 12, 196201, doi:10.11909/j.issn.1671-5411.2015.03.015 (2015).

71 Hill, R. D., Storandt, M. \& Malley, M. The impact of long-term exercise training on psychological function in older adults. J Gerontol 48, P12-17, doi:10.1093/geronj/48.1.p12 (1993).

72 Yeung, S. E. \& Loken Thornton, W. "Do it-yourself": Home blood pressure as a predictor of traditional and everyday cognition in older adults. PLoS One 12, e0177424, doi:10.1371/journal.pone.0177424 (2017).

73 Young, D. R., Appel, L. J., Jee, S. \& Miller, E. R., 3rd. The effects of aerobic exercise and T'ai Chi on blood pressure in older people: results of a randomized trial. J Am Geriatr Soc 47, 277-284, doi:10.1111/j.1532-5415.1999.tb02989.x (1999).

74 Ben Achour Lebib, S., Missaoui, B., Miri, I., Ben Salah, F. Z. \& Dziri, C. [Role of the Neurocom Balance Master in assessment of gait problems and risk of falling in elderly people]. Ann Readapt Med Phys 49, 210-217, doi:10.1016/j.annrmp.2006.03.005 (2006).

75 Lininger, M. R., Leahy, T. E., Haug, E. C. \& Bowman, T. G. Test-Retest Reliability of the Limits of Stability Test Performed by Young Adults Using Neurocom(R) Vsr Sport. Int J Sports Phys Ther 13, 800-807 (2018).

76 Clapp, W. C. \& Gazzaley, A. Distinct mechanisms for the impact of distraction and interruption on working memory in aging. Neurobiol Aging 33, 134-148, doi:S01974580(10)00042-4 [pii]

10.1016/j.neurobiolaging.2010.01.012 (2012).

77 Choi, S. D., Guo, L., Kang, D. \& Xiong, S. Exergame technology and interactive interventions for elderly fall prevention: A systematic literature review. Appl Ergon 65, 570-581, doi:10.1016/j.apergo.2016.10.013 (2017).

78 Maillot, P., Perrot, A., Hartley, A. \& Do, M. C. The braking force in walking: age-related differences and improvement in older adults with exergame training. J Aging Phys Act 22, 518-526, doi:10.1123/japa.2013-0001 (2014).

79 Rahe, J. et al. Effects of cognitive training with additional physical activity compared to pure cognitive training in healthy older adults. Clin Interv Aging 10, 297-310, doi:10.2147/CIA.S74071 (2015).

80 Davis, N. O., Bower, J. \& Kollins, S. H. Proof-of-concept study of an at-home, engaging, digital intervention for pediatric ADHD. PLoS One 13, e0189749, doi:10.1371/journal.pone.0189749 (2018).

81 Kollins, S. H. et al. A novel digital intervention for actively reducing severity of paediatric ADHD (STARS-ADHD): a randomised controlled trial. The Lancet Digital Health, doi:https://doi.org/10.1016/S2589-7500(20)30017-0 (2020).

82 Anguera, J. A. et al. A pilot study to determine the feasibility of enhancing cognitive abilities in children with sensory processing dysfunction. PLoS One 12, e0172616, doi:10.1371/journal.pone.0172616 (2017).

83 Gavelin, H. M. et al. Combined physical and cognitive training for older adults with and without cognitive impairment: A systematic review and network meta-analysis of randomized controlled trials. Ageing Res Rev 66, 101232, doi:10.1016/j.arr.2020.101232 (2021).

84 Lauenroth, A., loannidis, A. E. \& Teichmann, B. Influence of combined physical and cognitive training on cognition: a systematic review. BMC Geriatr 16, 141, doi:10.1186/s12877-016-0315-1 (2016).

85 Law, L. L., Barnett, F., Yau, M. K. \& Gray, M. A. Effects of combined cognitive and exercise interventions on cognition in older adults with and without cognitive impairment: a systematic review. Ageing Res Rev 15, 61-75, doi:10.1016/j.arr.2014.02.008 (2014). 

effects of combined cognitive and physical intervention on cognition in healthy older adults. Ageing Res Rev 31, 67-79, doi:10.1016/j.arr.2016.07.003 (2016).

87 Zheng, Z. et al. Combined cognitive-psychological-physical intervention induces reorganization of intrinsic functional brain architecture in older adults. Neural Plast 2015, 713104, doi:10.1155/2015/713104 (2015).

$88 \mathrm{Li}, \mathrm{R}$. et al. Multimodal intervention in older adults improves resting-state functional connectivity between the medial prefrontal cortex and medial temporal lobe. Front Aging Neurosci 6, 39, doi:10.3389/fnagi.2014.00039 (2014).

89 Schmiedt-Fehr, C. \& Basar-Eroglu, C. Event-related delta and theta brain oscillations reflect age-related changes in both a general and a specific neuronal inhibitory mechanism. Clin Neurophysiol 122, 1156-1167, doi:10.1016/j.clinph.2010.10.045 (2011).

90 Cabeza, R., Anderson, N. D., Locantore, J. K. \& Mclntosh, A. R. Aging gracefully: compensatory brain activity in high-performing older adults. Neuroimage 17, 1394-1402 (2002).

91 Reuter-Lorenz, P. A. \& Park, D. C. How does it STAC up? Revisiting the scaffolding theory of aging and cognition. Neuropsychol Rev 24, 355-370, doi:10.1007/s11065-0149270-9 (2014).

92 de Villers-Sidani, E. \& Merzenich, M. M. Lifelong plasticity in the rat auditory cortex: basic mechanisms and role of sensory experience. Prog Brain Res 191, 119-131, doi:10.1016/B978-0-444-53752-2.00009-6 (2011).

93 Ball, K. K., Beard, B. L., Roenker, D. L., Miller, R. L. \& Griggs, D. S. Age and visual search: expanding the useful field of view. J Opt Soc Am A 5, 2210-2219 (1988).

94 Willis, S. L. et al. Long-term effects of cognitive training on everyday functional outcomes in older adults. JAMA 296, 2805-2814, doi:296/23/2805 [pii]

10.1001/jama.296.23.2805 (2006).

95 Sandberg, P. \& Stigsdotter Neely, A. Long-term effects of executive process training in young and old adults. Neuropsychological rehabilitation 26, 761-782, doi:10.1080/09602011.2015.1108205 (2016).

96 Li, S. C. et al. Working memory plasticity in old age: practice gain, transfer, and maintenance. Psychol Aging 23, 731-742, doi:10.1037/a0014343 (2008).

97 Brehmer, Y. et al. Comparing memory skill maintenance across the life span: preservation in adults, increase in children. Psychol Aging 23, 227-238, doi:10.1037/0882-7974.23.2.227 (2008).

98 Borella, E., Carretti, B., Zanoni, G., Zavagnin, M. \& De Beni, R. Working memory training in old age: an examination of transfer and maintenance effects. Arch Clin Neuropsychol 28, 331-347, doi:10.1093/arclin/act020 (2013).

99 Dahlin, E., Nyberg, L., Backman, L. \& Neely, A. S. Plasticity of executive functioning in young and older adults: immediate training gains, transfer, and long-term maintenance.

Psychol Aging 23, 720-730, doi:2008-19072-005 [pii]

10.1037/a0014296 (2008).

100 Jurigova, B. G., Gerdes, M. R., Anguera, J. A. \& Marco, E. J. Sustained benefits of cognitive training in children with inattention, three-year follow-up. PLoS One 16, e0246449, doi:10.1371/journal.pone.0246449 (2021).

101 Salthouse, T. A. Mental Exercise and Mental Aging: Evaluating the Validity of the "Use It or Lose It" Hypothesis. Perspect Psychol Sci 1, 68-87, doi:10.1111/j.17456916.2006.00005.x (2006).

102 Rebok, G. W., Carlson, M. C. \& Langbaum, J. B. Training and maintaining memory abilities in healthy older adults: traditional and novel approaches. J Gerontol B Psychol Sci Soc Sci 62 Spec No 1, 53-61, doi:10.1093/geronb/62.special_issue_1.53 (2007).

103 Gheysen, F. et al. Physical activity to improve cognition in older adults: can physical activity programs enriched with cognitive challenges enhance the effects? A systematic 
review and meta-analysis. Int J Behav Nutr Phys Act 15, 63, doi:10.1186/s12966-0180697-x (2018).

104 Karssemeijer, E. G. A. et al. Positive effects of combined cognitive and physical exercise training on cognitive function in older adults with mild cognitive impairment or dementia: A meta-analysis. Ageing Res Rev 40, 75-83, doi:10.1016/j.arr.2017.09.003 (2017).

105 Colcombe, S. J. et al. Cardiovascular fitness, cortical plasticity, and aging. Proc Natl Acad Sci U S A 101, 3316-3321, doi:10.1073/pnas.0400266101

0400266101 [pii] (2004).

106 Voss, M. W. et al. Plasticity of brain networks in a randomized intervention trial of exercise training in older adults. Front Aging Neurosci 2, doi:10.3389/fnagi.2010.00032 (2010).

107 Oberauer, K. Working Memory and Attention - A Conceptual Analysis and Review. J Cogn 2, 36, doi:10.5334/joc.58 (2019).

108 Wollesen, B., Schulz, S., Seydell, L. \& Delbaere, K. Does dual task training improve walking performance of older adults with concern of falling? BMC Geriatr 17, 213, doi:10.1186/s12877-017-0610-5 (2017).

109 Emmanouel, A., Kontrafouri, E., Nikolaos, P., Kessels, R. P. C. \& Fasotti, L. Incorporation of a working memory strategy in GMT to facilitate serial-order behaviour in brain-injured patients. Neuropsychological rehabilitation 30, 888-914, doi:10.1080/09602011.2018.1517369 (2020).

110 Boot, W. R., Simons, D. J., Stothart, C. \& Stutts, C. The pervasive problem with placebos in psychology why active control groups are not sufficient to rule out placebo effects. Perspectives on Psychological Science 8, 445-454 (2013).

111 McAuley, T., Yap, M., Christ, S. E. \& White, D. A. Revisiting inhibitory control across the life span: insights from the ex-Gaussian distribution. Dev Neuropsychol 29, 447-458, doi:10.1207/s15326942dn2903_4 (2006).

112 Moret-Tatay, C. et al. The effects of age and emotional valence on recognition memory: an ex-Gaussian components analysis. Scand J Psychol 55, 420-426, doi:10.1111/sjop.12136 (2014).

113 Schmiedek, F., Oberauer, K., Wilhelm, O., Suss, H. M. \& Wittmann, W. W. Individual differences in components of reaction time distributions and their relations to working memory and intelligence. J Exp Psychol Gen 136, 414-429, doi:10.1037/00963445.136.3.414 (2007).

114 Vaurio, R. G., Simmonds, D. J. \& Mostofsky, S. H. Increased intra-individual reaction time variability in attention-deficit/hyperactivity disorder across response inhibition tasks with different cognitive demands. Neuropsychologia 47, 2389-2396, doi:10.1016/j.neuropsychologia.2009.01.022 (2009).

115 Cai, W. et al. Latent brain state dynamics distinguish behavioral variability, impaired decision-making, and inattention. Mol Psychiatry, doi:10.1038/s41380-021-01022-3 (2021).

116 Hommel, B., Li, K. Z. \& Li, S. C. Visual search across the life span. Dev Psychol 40, 545558, doi:10.1037/0012-1649.40.4.545 (2004).

117 Vogel, E. K., McCollough, A. W. \& Machizawa, M. G. Neural measures reveal individual differences in controlling access to working memory. Nature 438, 500-503, doi:nature04171 [pii]

10.1038/nature04171 (2005).

118 Jost, K., Bryck, R. L., Vogel, E. K. \& Mayr, U. Are old adults just like low working memory young adults? Filtering efficiency and age differences in visual working memory. Cereb Cortex 21, 1147-1154, doi:10.1093/cercor/bhq185 (2011).

119 Lee, E. Y. et al. Visual working memory deficits in patients with Parkinson's disease are due to both reduced storage capacity and impaired ability to filter out irrelevant information. Brain 133, 2677-2689, doi:10.1093/brain/awq197 (2010). 
120 Delorme, A. \& Makeig, S. EEGLAB: an open source toolbox for analysis of single-trial EEG dynamics including independent component analysis. J Neurosci Methods. 134, 921. doi: 10.1016/j.jneumeth.2003.1010.1009. (2004).

121 Lutz, A. et al. Mental training enhances attentional stability: neural and behavioral evidence. The Journal of neuroscience : the official journal of the Society for Neuroscience 29, 13418-13427 (2009).

122 Clayton, M. S., Yeung, N. \& Cohen Kadosh, R. The roles of cortical oscillations in sustained attention. Trends Cogn Sci. 19, 188-195. doi: 110.1016/j.tics.2015.1002.1004. Epub 2015 Mar 1019. (2015).

123 Polich, J. Updating P300: an integrative theory of P3a and P3b. Clin Neurophysiol. 118, 2128-2148. doi: 2110.1016/j.clinph.2007.2104.2019. Epub 2007 Jun 2118. (2007).

124 Papenberg, G., Hammerer, D., Muller, V., Lindenberger, U. \& Li, S. C. Lower theta intertrial phase coherence during performance monitoring is related to higher reaction time variability: a lifespan study. Neuroimage $\mathbf{8 3}, 912-920$, doi:10.1016/j.neuroimage.2013.07.032 (2013).

125 DeMers, D. \& Wachs, D. in StatPearls (2021).

126 Sesso, H. D. et al. Systolic and diastolic blood pressure, pulse pressure, and mean arterial pressure as predictors of cardiovascular disease risk in Men. Hypertension 36, 801-807, doi:10.1161/01.hyp.36.5.801 (2000).

127 Yu, D., Zhao, Z. \& Simmons, D. Interaction between Mean Arterial Pressure and HbA1c in Prediction of Cardiovascular Disease Hospitalisation: A Population-Based CaseControl Study. J Diabetes Res 2016, 8714745, doi:10.1155/2016/8714745 (2016).

128 Miura, K. et al. Pulse pressure compared with other blood pressure indexes in the prediction of 25-year cardiovascular and all-cause mortality rates: The Chicago Heart Association Detection Project in Industry Study. Hypertension 38, 232-237, doi:10.1161/01.hyp.38.2.232 (2001).

129 Jones, C. J. \& Rikli, R. E. Measuring functional fitness of older adults. The Journal on active aging 1, 24-30 (2002).

130 Khammar, A., Yarahmadi, M. \& Madadizadeh, F. What Is Analysis of Covariance (ANCOVA) and How to Correctly Report Its Results in Medical Research? Iran J Public Health 49, 1016-1017 (2020).

131 Hedges, L. V. \& Olkin, I. Statistical methods for meta-analysis. (Academic Press, 1985).

132 Norris, E., Hubbuch, E., Ford, A. \& Allen, W. The relationship of weight-bearing and nonweight bearing ankle dorsiflexion to balance and gait performance in young and older adults. Physical Therapy and Rehabilitation. 3.6, doi:10.7243/2055-2386-3-6. (2016).

133 International, N. (Clackamas, OR, 2001).

134 Pickerill, M. L. \& Harter, R. A. Validity and reliability of limits-of-stability testing: a comparison of 2 postural stability evaluation devices. J Athl Train 46, 600-606, doi:10.4085/1062-6050-46.6.600 (2011).

135 Kelley, G. A., Kelley, K. A. \& Tran, Z. V. Aerobic exercise and resting blood pressure: a meta-analytic review of randomized, controlled trials. Prev Cardiol 4, 73-80, doi:10.1111/j.1520-037x.2001.00529.x (2001).

136 Kelley, G. A. \& Sharpe Kelley, K. Aerobic exercise and resting blood pressure in older adults: a meta-analytic review of randomized controlled trials. J Gerontol A Biol Sci Med Sci 56, M298-303, doi:10.1093/gerona/56.5.m298 (2001). 
Table 1. Group means and standard deviations for each primary measure at each timepoint for each group.

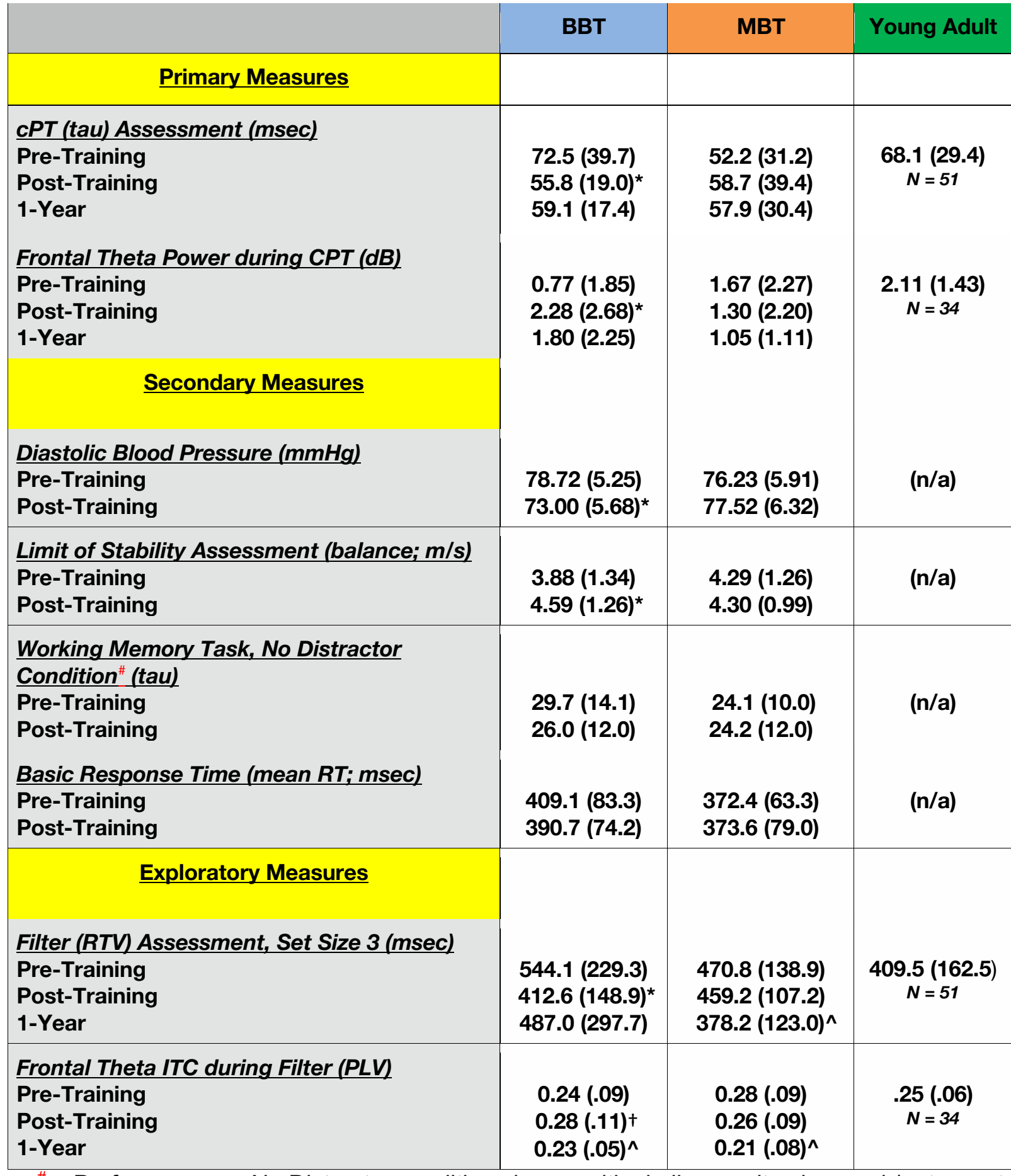

\# = Performance on No Distractor condition shown, with similar results observed (not reported) on the Attend Distractor and Ignore Distractor conditions.

${ }^{*}=$ Post-training performance significantly different than Pre-training performance $(+=p=.08)$

$\wedge=$ Post-training performance significantly different than 1-Year performance

Note that no significant difference at baseline was observed between groups (see Statistics for details). 


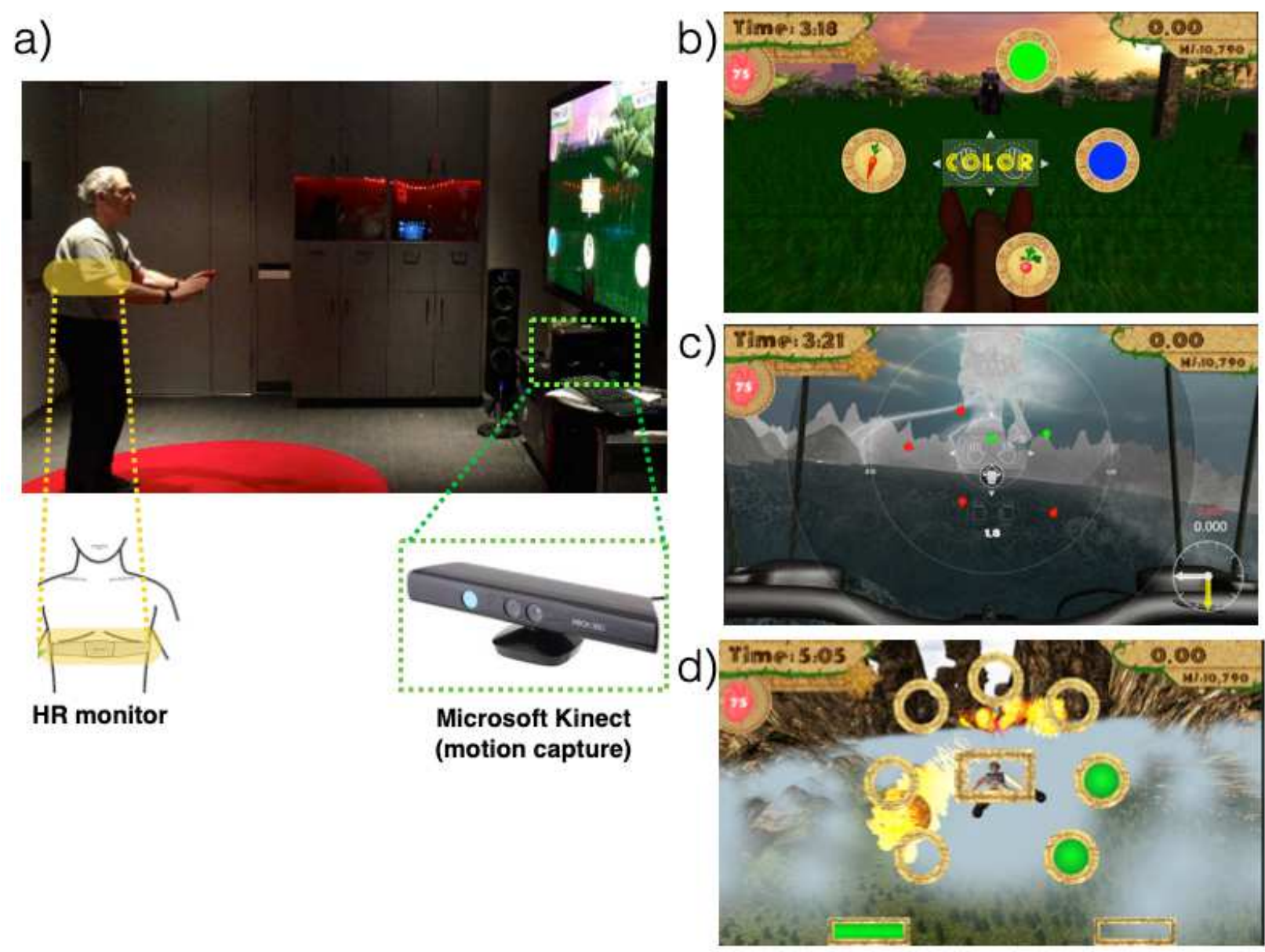

Figure 1. Body-Brain Trainer (BBT) platform. a, Image of participant playing BBT. Highlighted is the use of a heart rate monitor to assess and adapt the physical intensity of game play in real-time, as well as the Microsoft Kinect ${ }^{\mathrm{TM}}$ motion capture technology used to collect responses with one's hands and/or feet based on the cognitive task presented on the monitor, and adapt the cognitive difficulty of each game in real-time. b, Image of the task switching module. c, Image of the attention module. d, Image of the working memory module. 
a)

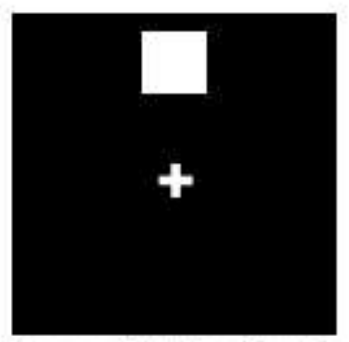

Respond to the stimulus

b) CPT Task (Pre - Post) Scores

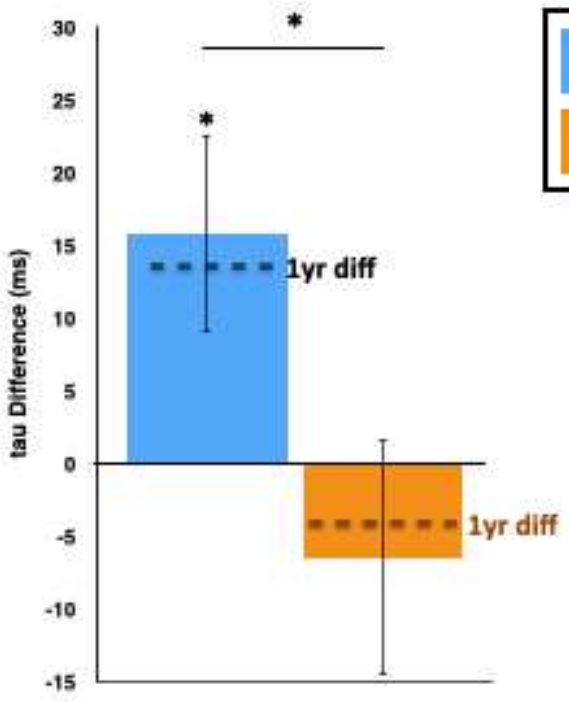

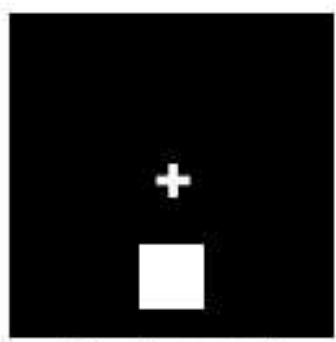

Do not respond
C) CPT Task mfTheta Power (Post - Pre) Scores

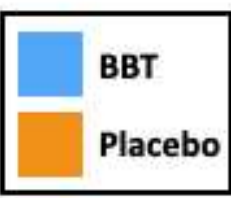

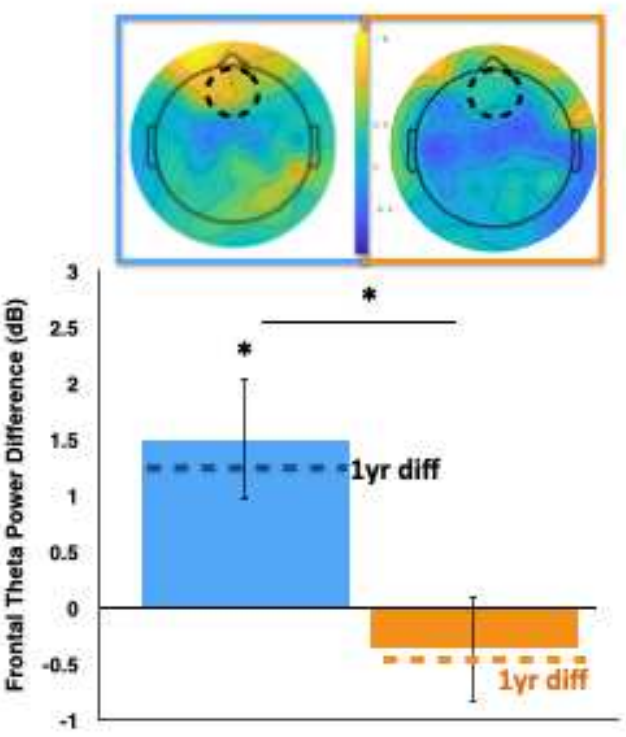

Figure 2. CPT task. a, Stimuli and protocol for the attention without distraction (CPT) task. b, Bar graphs illustrating the group mean change in ex-gaussian tau (pre - post, with + values demonstrating improvement in tau over time) for each group, with the dashed line (1-year diff = pre - 1-year) illustrating the change in tau at the 1-year mark. c, Bar graphs and topographic plots illustrating the group mean change in midline frontal theta power (post - pre) for each group, with the dashed line on the bar graphs illustrating the change in power at the 1-year mark (1-year - pre). The dashed circle on the topographic plot illustrates the electrodes where statistical analyses took place. ${ }^{*}=p<.05$. Error bars represent s.e.m. 
Figure 3. Comparison of BBT and MBT performance versus younger adults.

\begin{tabular}{|c|c|c|c|c|}
\hline & $\begin{array}{c}\text { BBT } \\
\text { Pre-Training }\end{array}$ & $\begin{array}{c}\text { MBT } \\
\text { Pre-Training }\end{array}$ & $\begin{array}{c}\text { BBT } \\
\text { Post-Training }\end{array}$ & $\begin{array}{c}\text { MBT } \\
\text { Post-Training }\end{array}$ \\
\hline $\begin{array}{c}\text { CPT (tau) } \\
\text { CPT } \\
\text { (mfTheta Power) }\end{array}$ & - & - & & $=$ \\
\hline
\end{tabular}

$\downarrow$ => performance significantly lesser than that of young adults

1 => performance significantly better than that of young adults

$=$ => performance equivalent to that of young adults

Green = Significant improvement from pre-training

Red $=$ Significant decline from pre-training 
a.

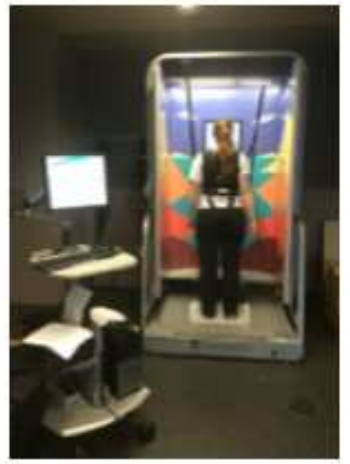

b. Limits of Stability (Post-Pre) Scores

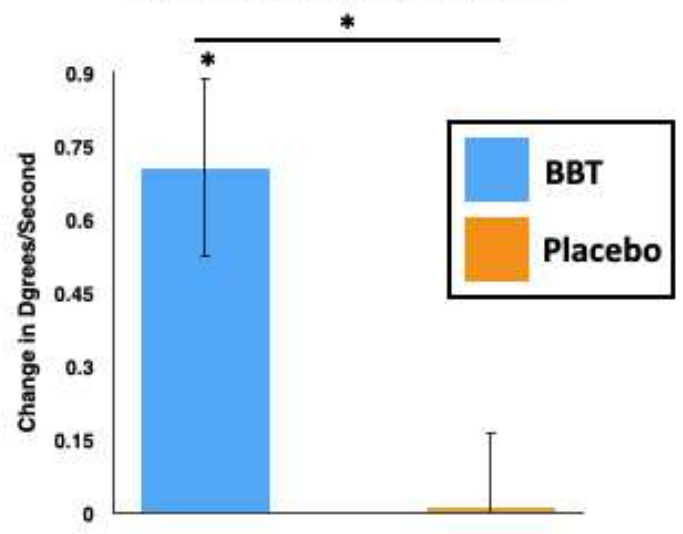

C.

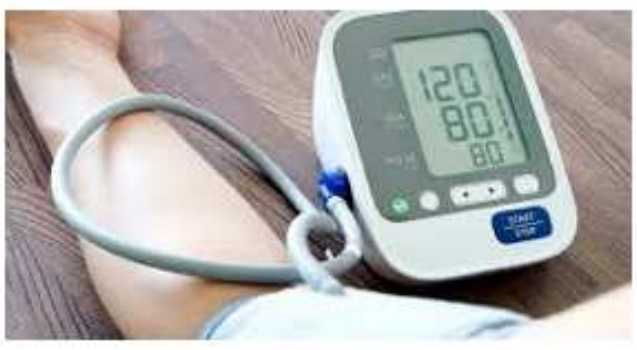

d. Diastolic Blood Pressure (Pre-Post) Scores

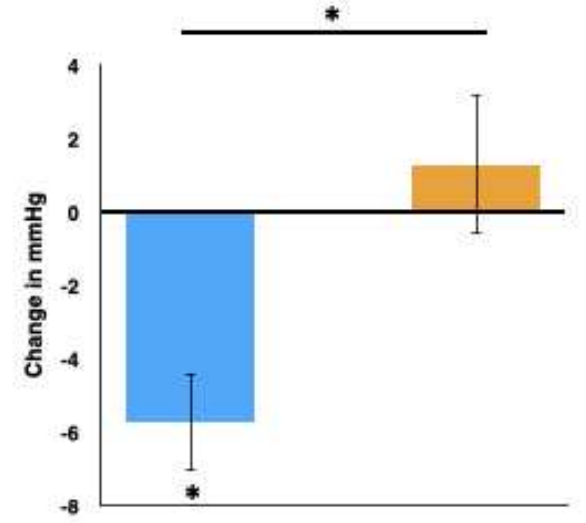

\section{Figure 4. Improvements in Physical Outcomes Assessments}

a, Limits of Stability (LoS) assessment on the NeuroCom balance manager system ${ }^{132,133}$. The LoS is a center-out postural stability test that serves as a basic balance quantification metric. During this task, increases in movement velocity have been associated with decreases in fall risk in older adults ${ }^{134} . b$, There was a significant group $x$ time interaction in movement velocity of the LoS task $\left(F_{1,40}=7.814, p=0.008\right.$, partial eta squared $\left.=0.163\right)$. The BBT group showed a significant increase from pre to post $\left(t_{18}=4.53, p<0.001\right)$, whereas the control participants showed no difference $\left(t_{22}=-1.02, p=0.32\right)$. Note there was no difference at baseline between groups $\left(t_{43}=1.77, p=.08\right.$. $) c$, Diastolic blood pressure assessment. Diastolic blood pressure is a predictor of overall physical health and has been found to be decreased through exercise interventions ${ }^{135,136}$. $d$, There was a significant group $x$ time interaction $\left(F_{1,40}=8.43, p=0.006\right)$. The BBT group showed a significant decrease from pre to post $\left(t_{19}=4.31, p<0.001\right)$, whereas the control participants showed no significant change following their intervention $\left(t_{21}=0.46, p=0.65\right)$. Note there was no difference at baseline between groups $\left(t_{44}=1.05, p=.30\right)$. 


\section{Supplementary Files}

This is a list of supplementary files associated with this preprint. Click to download.

- AngueraBBTLIFESPANSupplementaryMaterialsV4.pdf 